\title{
Variational Principles for Maximization Problems with Lower-semicontinuous Goal Functions
}

\author{
M. Ivanov ${ }^{1}$ · P.S. Kenderov ${ }^{2}$ (D) . J.P. Revalski ${ }^{2,3}$
}

Received: 20 July 2020 / Accepted: 2 August 2021 /Published online: 20 September 2021

(C) The Author(s) 2021

\section{Abstract}

Let $X$ be a completely regular topological space and $f$ a real-valued bounded from above lower semicontinuous function in it. Let $C(X)$ be the space of all bounded continuous realvalued functions in $X$ endowed with the usual sup-norm. We show that the following two properties are equivalent:

(a) $X$ is $\alpha$-favourable (in the sense of the Banach-Mazur game);

(b) The set of functions $h$ in $C(X)$ for which $f+h$ attains its supremum in $X$ contains a dense and $G_{\delta}$-subset of the space $C(X)$.

In particular, property (b) has place if $X$ is a compact space or, more generally, if $X$ is homeomorphic to a dense $G_{\delta}$ subset of a compact space.

We show also the equivalence of the following stronger properties:

(a') $X$ contains some dense completely metrizable subset;

$\left(\mathrm{b}^{\prime}\right)$ the set of functions $h$ in $C(X)$ for which $f+h$ has strong maximum in $X$ contains a dense and $G_{\delta}$-subset of the space $C(X)$.

If $X$ is a complete metric space and $f$ is bounded, then the set of functions $h$ from $C(X)$ for which $f+h$ has both strong maximum and strong minimum in $X$ contains a dense $G_{\delta}$-subset of $C(X)$.

Keywords Variational principle in optimization · Topological game $\cdot$ Strong maximum · Fragmentable sets

Mathematics Subject Classification (2010) 49J27 · 54E52 · 54C60 • 49J53

The first and second author were partially supported by the Bulgarian National Fund for Scientific Research, under grant KP-06-H22/4; The third author was partially supported by the Grant No BG05M2OP001-1.001-0003, financed by the Science and Education for Smart Growth Operational Program (2014-2020) and co-financed by the European Union through the European structural and Investment funds.

P.S. Kenderov

kenderovp@cc.bas.bg

Extended author information available on the last page of the article. 


\section{Introduction}

Let $X$ be a completely regular topological space and let $f: X \rightarrow \mathbb{R}$ be a real-valued lower semicontinuous function which is bounded from below. A typical variational principle in optimization theory gives conditions on $X$ under which the function $f$ can be perturbed by some other function $h$ from a prescribed set $Y$ of functions so that $f+h$ attains its infimum on $X$. Very often these principles establish that the set of such "good perturbations" is rather big in the prescribed set $Y$ of all perturbations. For example, it can be dense in $Y$ or even contain a dense and $G_{\delta}$-subset of $Y$ (in the latter case it is said that a Generic Variational Principle has place). The meaning of such variational principles is that a bad optimization problem (say, without a solution) can be slightly perturbed in order to get a better optimization problem (with solution). Examples of such principles are the Ekeland Variational Principle [10], the Smooth Variational Principles of Borwein-Preiss [1], DevilleGodefroy-Zizler Variational Principle [9], Stegall Variational Principle [17], the Generic Continuous Variational Principles established by Lucchetti and Patrone [15], De Blasi and Myiak [7] as well as the Variational Principles for general topological spaces proved in [2-4].

In contrast to this trend, in [11] the author considered the problem of maximizing a lower semicontinous function $f$ which is bounded from above and proved, using a direct approach, that, if $X$ is a complete metric space and $B U C(X)$ is the space of bounded uniformly continuous functions on $X$ (equipped with the sup-norm), then there exists a dense and $G_{\delta}$-subset $H \subset B U C(X)$ such that for each $h \in H$ the perturbed function $f+h$ attains its strong maximum in $X$ (the latter means that the function $f+h$ has a unique maximizer in $X$ towards which converges every maximizing sequence for $f+h)$. The goal of this paper is to prove generic Maximization Variational Principles for general (not necessarily metrizable) topological spaces $X$ and for functions $f$ more general than the lower semicontinuous ones. The perturbations are from the space $C(X)$ of all bounded continuous functions in $X$ which is equipped with the sup-norm. We give necessary and sufficient conditions for the validity of such variational principles in terms of existence of winning strategies for one of the players in the famous Banach-Mazur game played in $X$ (denoted by $B M(X)$ in the sequel). A partial case of our main result, Theorem 3.3, reads as follows:

For a given completely regular topological space $X$ the following assertions are equivalent:

(i) The $\alpha$-player has a winning strategy in the game $B M(X)$;

(ii) For every bounded from above lower semicontinuous function $f: X \rightarrow \mathbb{R}$ the set of perturbations $S(f):=\{h \in C(X): f+h$ attains it supremum in $X\}$ contains a dense and $G_{\delta}$-subset of $C(X)$;

(iii) There exists a bounded from above lower semicontinuous function $f: X \rightarrow \mathbb{R}$ for which the set $S(f)$ contains a dense and $G_{\delta}$-subset of $C(X)$.

In particular, Theorem 3.3 is valid for compact spaces $X$ (see Theorem 4.1) as well as for complete metric spaces $X$ (see Corollary 3.5). It generalizes Theorem 3.1 from [13], where the case $f \equiv 0$ was considered.

A characterization of the spaces $X$ for which a generic variational principle for strong maximums is valid is given in Corollary 3.5. A partial case of it says: 
For the completely regular topological space $X$ the following assertions are equivalent:

(i) For every bounded from above lower semicontinuous function $f: X \rightarrow \mathbb{R}$ the set $\{h \in C(X): f+h$ attains its strong maximum in $X\}$ contains a dense and $G_{\delta}$-subset of $C(X)$;

(ii) The space $X$ contains a dense completely metrizable subset.

Putting together Corollary 3.5 from this paper and Theorem 3.5 from [4] we get Corollary 4.4:

If $f$ is a bounded lower semicontinuous function defined in the complete metric space $X$, then the set $\{h \in C(X): f+h$ attains both its strong maximum and its strong minimum in $X\}$ contains a dense and $G_{\delta}$-subset of $C(X)$.

At the end of the introduction we would like to underline one essential difference between the variational principles for minimization problems and the variational principles for maximization problems considered in this paper. For every bounded from below lower semicontinuous function $f$ defined in an arbitrary completely regular topological space $X$ the set $\{h \in C(X): f+h$ attains its infimum in $X\}$ is a dense subset of $C(X)$ (Lemma 2.1 in [14]). This fact was in the base of the considerations in [4] where general generic variational principles for minimization problems were established. The situation with the variational principles for maximization problems is entirely different. As Example 2.4 below shows there exists a bounded from above lower semicontinuous function $f$ for which the set $\{h \in C(X): f+h$ attains its supremum in $X\}$ is empty. Such examples show that the approach used in [4] to provide generic variational principles for minimization problems is inappropriate for the considerations that follow.

The structure of the paper is simple. In the next section we give some necessary preliminaries and prove an auxiliary lemma about approximate maximizers which is essential for the proofs of our results. We briefly recall the concept of Banach-Mazur game and the corresponding notions of winning strategies for the players. The main results are presented in Section 3. In the final Section 4 we outline several applications.

\section{Some Preliminaries}

Let $f: X \rightarrow \mathbb{R}$ be a bounded from above real-valued function defined in the completely regular topological space $X$. Let $C(X)$ be the space of all real-valued bounded continuous functions in $X$, equipped, as usual, with the sup-norm $\|h\|_{\infty}:=\sup \{|h(x)|: x \in X\}$, $h \in C(X)$. With this norm $C(X)$ is a real Banach space.

Consider the set-valued mapping $S_{f}: C(X) \times \mathbb{R}_{++} \rightarrow X$ defined by

$$
S_{f}(h, \varepsilon):=\left\{x \in X:(f+h)(x)>\sup _{X}(f+h)-\varepsilon\right\}, \quad(h, \varepsilon) \in C(X) \times \mathbb{R}_{++},
$$

where $\mathbb{R}_{++}$is the set of positive numbers.

This mapping gives the $\varepsilon$-solutions to the maximization problem generated by the function $f+h$ in $X$. The mapping $S_{f}$ is always nonempty-valued and, when $f$ is lower semicontinuous, this mapping is also with open images. For a fixed $h \in C(X)$ the intersection of $S_{f}(h, \varepsilon)$ over all $\varepsilon>0$ gives exactly the set of maximizers of the function $f+h$ on $X$, which we denote by $M_{f}(h)$, that is

$$
M_{f}(h):=\left\{x \in X:(f+h)(x)=\sup _{X}(f+h)\right\}=\cap_{\varepsilon>0} S_{f}(h, \varepsilon), \quad h \in C(X) .
$$


In contrast to the mapping $S_{f}(\cdot, \cdot)$, the set-valued mapping $M_{f}: C(X) \rightrightarrows X$ may have empty images for many $h \in C(X)$ or even for all $h \in C(X)$ as the two examples after Remark 2.3 show.

The following lemma gives the properties of the mapping $S_{f}(\cdot, \cdot)$ that will be frequently used in the sequel. Below, as usual, $B(h, \varepsilon)$ (resp. $B[h, \varepsilon])$ denotes the open (resp. closed) ball in $C(X)$ centered at $h$ and with radius $\varepsilon>0$. For a set $A \subset C(X)$ and $\varepsilon>0$ we denote by $S_{f}(A, \varepsilon)$ the union of all $S_{f}(h, \varepsilon), h \in A$.

Lemma 2.1 Suppose that $f: X \rightarrow \mathbb{R}$ is a bounded from above real-valued function defined in the completely regular space $X$. Then:

(a) if $\left(B_{n}\right)_{n}$ is a nested sequence of sets in $C(X)$, such that $\operatorname{diam}\left(B_{n}\right) \rightarrow 0,\left\{h_{0}\right\}:=\cap_{n} B_{n}$ for some $h_{0} \in C(X)$, and $\left(\delta_{n}\right)_{n}$ is a sequence of positive integers such that $\delta_{n} \rightarrow 0$, then $M_{f}\left(h_{0}\right)=\cap_{n} S_{f}\left(B_{n}, \delta_{n}\right)$;

(b) let $V$ be a nonempty open subset of $X$ such that $V \cap S_{f}\left(h_{0}, \varepsilon\right) \neq \emptyset$ for some $h_{0} \in$ $C(X)$ and $\varepsilon>0$. Then there exists $h_{1} \in C(X)$ and $\delta>0, \delta \leq \varepsilon / 4$, such that $B\left(h_{1}, \delta / 2\right) \subset B\left(h_{0}, \varepsilon\right)$ and $S_{f}\left(B\left(h_{1}, \delta / 2\right), \delta\right) \subset V$.

Proof (a) Let $\left(B_{n}\right)_{n}, h_{0}$ and $\left(\delta_{n}\right)_{n}$ be as in the lemma. Obviously $M_{f}\left(h_{0}\right) \subset \cap_{n} S_{f}\left(B_{n}, \delta_{n}\right)$. Suppose that $x \in \cap_{n} S_{f}\left(B_{n}, \delta_{n}\right)$. Then, there is a sequence $\left(h_{n}\right)_{n} \subset C(X)$ such that for each $n \geq 1$ we have $h_{n} \in B_{n}$ (which implies $\left\|h_{n}-h_{0}\right\|_{\infty} \rightarrow 0$ ) and, moreover,

$$
f(x)+h_{n}(x)>\sup _{X}\left(f+h_{n}\right)-\delta_{n} .
$$

The latter yields (passing to the limit, and having in mind that $h_{n}$ uniformly converges to $h_{0}$ ) that $x$ is a maximum point for $f+h_{0}$ in $X$, that is $x \in M_{f}\left(h_{0}\right)$. The proof of the assertion (a) is completed.

(b) Take some $x_{0} \in V \cap S_{f}\left(h_{0}, \varepsilon\right)$ and consider $\bar{h} \in C(X)$ such that $0 \leq \bar{h}(x) \leq 1$ for every $x \in X, \bar{h}\left(x_{0}\right)=1$ and $\left.\bar{h}\right|_{X \backslash V} \equiv 0$. Since $x_{0} \in S_{f}\left(h_{0}, \varepsilon\right)$ we have, on the one side,

$$
\left(f+h_{0}+\varepsilon \bar{h}\right)\left(x_{0}\right)=f\left(x_{0}\right)+h_{0}\left(x_{0}\right)+\varepsilon>\sup _{X}\left(f+h_{0}\right) .
$$

On the other side, note that

$$
\sup _{X}\left(f+h_{0}+\varepsilon \bar{h}\right) \leq \sup _{X}\left(f+h_{0}\right)+\sup _{X} \varepsilon \bar{h}=\sup _{X}\left(f+h_{0}\right)+\varepsilon .
$$

Set now $\delta:=\left(\left(f+h_{0}+\varepsilon \bar{h}\right)\left(x_{0}\right)-\sup _{X}\left(f+h_{0}\right)\right) / 4$ and observe that, according to the first inequality above, $\delta>0$ and, according to the second,

$$
\delta=\frac{1}{4}\left(\left(f+h_{0}+\varepsilon \bar{h}\right)\left(x_{0}\right)-\sup _{X}\left(f+h_{0}\right)\right) \leq \frac{1}{4}\left(\sup _{X}\left(f+h_{0}+\varepsilon \bar{h}\right)-\sup _{X}\left(f+h_{0}\right)\right) \leq \frac{1}{4} \varepsilon .
$$

Put $h^{*}:=\varepsilon \bar{h}$ and let $h \in C(X)$ be such that $\left\|h-h^{*}\right\|_{\infty}<\delta$. For every $x \in S_{f}\left(h_{0}+h, \delta\right)$ we have, on the one hand,

$$
\begin{aligned}
\left(f+h_{0}+h\right)(x) & >\sup _{X}\left(f+h_{0}+h\right)-\delta \geq\left(f+h_{0}+h\right)\left(x_{0}\right)-\delta \\
& =\left(f+h_{0}+h^{*}\right)\left(x_{0}\right)+\left(h-h^{*}\right)\left(x_{0}\right)-\delta \\
& =4 \delta+\sup _{X}\left(f+h_{0}\right)+\left(h-h^{*}\right)\left(x_{0}\right)-\delta \\
& \geq 4 \delta+\sup _{X}\left(f+h_{0}\right)-\delta-\delta=2 \delta+\sup _{X}\left(f+h_{0}\right) .
\end{aligned}
$$


On the other hand, for $x \notin V$ we have $h^{*}(x)=0$ and therefore,

$$
\begin{aligned}
\left(f+h_{0}+h\right)(x) & =\left(f+h_{0}+h^{*}\right)(x)+\left(h-h^{*}\right)(x) \\
& =\left(f+h_{0}\right)(x)+\left(h-h^{*}\right)(x) \\
& \leq\left(f+h_{0}\right)(x)+\delta \leq \sup _{X}\left(f+h_{0}\right)+\delta .
\end{aligned}
$$

Combining the last two chains of inequalities and equalities we conclude that $S_{f}\left(h_{0}+h, \delta\right) \subset V$ for every $h \in C(X)$ with $\left\|h-h^{*}\right\|_{\infty}<\delta$.

Consider finally, the element $h_{1}$ on the segment $\left[h_{0}, h_{0}+h^{*}\right]$ which is at distance $\delta / 2$ from $h_{0}+h^{*}$. Evidently, the ball $B\left(h_{1}, \delta / 2\right)$ is included both in $B\left(h_{0}, \varepsilon\right)$ and $B\left(h_{0}+h^{*}, \delta\right)$. And this completes the proof of the assertion (b).

Two remarks are in order here.

Remark 2.2 A close look at the proof of the assertions (a) and (b) above shows that if $Z$ is a linear subspace of $C(X)$ (or, more generally, if $Z$ is a convex cone in $C(X)$ ) such that for every open set $V$ of $X$ and $x \in V$ there exists an element $h \in Z$, such that $0 \leq h(y) \leq 1$ for every $y \in X, h(x)=1$ and $\left.h\right|_{X \backslash V} \equiv 0$, then the assertions (a) and (b) from Lemma 2.1 are true restricted to $Z$.

Remark 2.3 Though the above lemma is valid for arbitrary bounded from above functions one cannot expect to have meaningful variational principles without additional requirements imposed both on the function $f$ and on the space $X$. The following two examples illustrate this.

Example 2.4 Let $X$ be the set of all rational numbers with the topology inherited from the real line. Enumerate the elements of $X$ as a sequence $\left(r_{n}\right)_{n}$ and consider the function $f\left(r_{n}\right)=-n^{-1}$ which is bounded from above and lower semicontinuous. For every $h \in$ $C(X)$ and $r_{k} \in X$ one can find $r_{l} \in X$ such that $(f+h)\left(r_{k}\right)<(f+h)\left(r_{l}\right)$. Therefore $M_{f}(h)=\emptyset$ for every $h \in C(X)$ and, consequently, $S(f)=\emptyset$.

Example 2.5 Let $X$ be the unit interval [0,1]. Enumerate as above the rational numbers from this interval as a sequence $\left(r_{n}\right)_{n}$ and define the function $f$ to be zero at every irrational number and $f\left(r_{n}\right)=1-n^{-1}$ for every rational number $r_{n}$ from the sequence. It is easy to realize that no perturbed function $f+h$, where $h \in C([0,1])$, attains its supremum in $X$. I.e. $S(f)=\emptyset$.

At the end of this section, we remind two versions of the famous Banach-Mazur game which will be played correspondingly in $X$ and in $C(X)$. In the space $X$ we will use the most popular version of the Banach-Mazur game. It runs as follows. Two players, $\alpha$ and $\beta$, chose one after another nonempty open subsets of $X$. Player $\beta$ starts by choosing a nonempty open set $U_{1}$ of $X$ and player $\alpha$ responds by choosing a nonempty open set $V_{1}$ such that $V_{1} \subset U_{1}$. Then, $\beta$ continues by choosing a nonempty open set $U_{2}$ of $X$ included in $V_{1}$ and $\alpha$ chooses a nonempty open set $V_{2} \subset U_{2}$. In the next moves each player chooses an open nonempty subset of the other player's choice. As a result an infinite sequence of sets $\left\{U_{n}, V_{n}\right\}_{n}$ is generated which is called a play in this game. Each play satisfies the requirement $U_{n+1} \subset V_{n} \subset U_{n}$ for every $n \geq 1$. By definition, the player $\alpha$ wins the play $\left\{U_{n}, V_{n}\right\}_{n}$ if $\cap_{n} U_{n}=\cap_{n} V_{n} \neq \emptyset$. Otherwise, the player $\beta$ wins this play. We denote this game by $B M(X)$. 
Any finite sequence $\left(U_{1}, V_{1}, \ldots, U_{n}\right)$ or $\left(U_{1}, V_{1}, \ldots, U_{n}, V_{n}\right)$ in which the choices of the two players are made according to the rules of the game $B M(X)$ is called a partial play in this game. A strategy $s$ for the player $\alpha$ is a rule which assigns to each partial play $\left(U_{1}, V_{1}, \ldots, U_{n}\right)$ appearing in the course of the game a nonempty open set $V_{n}:=$ $s\left(U_{1}, V_{1}, \ldots, U_{n}\right)$. The play $\left\{U_{n}, V_{n}\right\}_{n}$ is called an $s$-play if every $V_{n}$ is chosen by using the strategy $s$. The strategy $s$ is called winning for the player $\alpha$ if every $s$-play $\left\{U_{n}, V_{n}\right\}_{n}$ is won by player $\alpha$.

The second version of the Banach-Mazur game (which, in fact, is the original version of this game - see Problem 43 in the book of Ulam [18]) will be played in $C(X)$ and denoted by $B M(C(X), S)$, where $S$ is some subset of $C(X)$. Two players, Player I and Player II, chose one after another nonempty open subsets of $C(X)$. The choices of Player I (who starts the game) are denoted by $W_{n}$. The choices of Player II are denoted by $W_{n}^{\prime}$. Choices are subjected to the requirement that $W_{n+1} \subset W_{n}^{\prime} \subset W_{n}$ for every $n \geq 1$. Player II wins the play $\left\{W_{n}, W_{n}^{\prime}\right\}_{n}$ if $\cap W_{n}=\cap W_{n}^{\prime} \subset S$. Otherwise, Player I wins this play. The notions of partial play and (winning) strategy for the Player II is defined as in the game $B M(X)$. According to a result from the book of Oxtoby [16], Player II has a winning strategy in this game if and only if the set $S$ contains a dense and $G_{\delta}$-subset of $C(X)$

\section{Existence of Solutions to Perturbed Maximisation Problems for Quasi Lower Semicontinuous Functions}

As stated in the title and in the introduction we are primarily interested in maximization variational principles for lower semicontinuous functions $f$. All of the results however are valid for functions $f$ which satisfy the following less restrictive condition obtained by a mild modification of the usual definition of lower semicontinuity.

Definition 3.1 The real valued function $f: X \rightarrow \mathbb{R}$ is called quasi lower semicontinuous at $x_{0} \in X$ if, for every $\varepsilon>0$, there exists an open set $U \subset X$ such that $x_{0} \in \bar{U}$ (the closure of $U$ ) and $f(x)>f\left(x_{0}\right)-\varepsilon$ for every $x \in U . f$ is said to be quasi lower semicontinuous (qlsc) if it is quasi lover semicontinuous at every $x \in X$.

If the requirement $x_{0} \in \bar{U}$ is replaced by the stronger one $x_{0} \in U$, we get the usual definition of lover semi continuity at $x_{0}$. The justification for the use of the word "quasi" in this context comes from a paper of Kempisty [12] where the term "quasicontinuous function" is used for functions with the following property: for every $x \in X$ and $\varepsilon>0$, there is an open subset $U$ of $X$ such that $x \in \bar{U}$ and $|f(y)-f(x)|<\varepsilon$ for every $y \in U$. Here is a simple example of a function which is qlsc but not lsc:

Example 3.2 Let $f(x)=-1$ if $x<0, f(x)=1$ for $x>0$ and $f(0)=0$.

It is easy to check that $f: X \rightarrow \mathbb{R}$ is qlsc at $x \in X$ if and only if for every $\varepsilon>0$ and every open set $U$ containing $x$ there exists a nonempty open set $U^{\prime} \subset U$ such that $f(y)>f(x)-\varepsilon$ for each $y \in U^{\prime}$.

If $f$ is qlsc and $h \in C(X)$, then $f+h$ is qlsc as well. The qlsc functions satisfy the following condition which will be used in the proofs of our results:

$\left(^{*}\right)$ For every $h \in C(X)$ and $\varepsilon>0$ the set int $S_{f}(h, \varepsilon)$ (the interior of $S_{f}(h, \varepsilon)$ ) is not empty. 
It is possible to prove that condition $\left(^{*}\right)$, in its turn, implies that $f$ is qlsc. We omit the proof of this fact because we are not going to use it.

The lower semicontinuous functions $f$ evidently satisfy condition $\left(^{*}\right)$ because the set $S_{f}(h, \varepsilon)$ in this case is (nonempty and) open for every $h \in C(X)$ and every $\varepsilon>0$.

Before formulating our main result we would like to mention one more difference between maximization and minimization problems with lower semicontinuous goal functions $f$. The set of minimizers in $X$ for $f+h, h \in C(X)$, is always closed. As simple examples show, the set $M_{f}(h)$ of maximizers of $f+h, h \in C(X)$, is not obliged to be closed. For qlsc functions $f$ neither the set of maximizers nor the set of minimizers of $f+h, h \in C(X)$, is obliged to be closed. Nevertheless, we obtain in the next theorem a generic maximization variational principle in which the good perturbations not only assure existence of maximizers of the perturbed function, but also the set of such maximizers is closed.

Recall that by $M_{f}(h)$ we denoted the set of maximizers of the function $f+h$ and by $S(f)$ the set of those $h \in C(X)$ such that the function $f+h$ attains its maximum in $X$. Let

$$
M(f):=\left\{h \in C(X): M_{f}(h) \text { is a closed subset of } X\right\} .
$$

Then we have:

Theorem 3.3 Let $X$ be a completely regular topological space. Then, the following statements are equivalent:

(a) There exists a winning strategy s for the player $\alpha$ in the game $B M(X)$;

(b) For every qlsc bounded from above function $f: X \rightarrow \mathbb{R}$ the set $S(f) \cap M(f)$ contains a dense and $G_{\delta}$-subset of $C(X)$.

(c) There exists a qlsc bounded from above function $f: X \rightarrow \mathbb{R}$ for which the set $S(f)$ contains a dense and $G_{\delta}$-subset of $C(X)$.

Proof $(a) \Rightarrow(b)$. Let $f: X \rightarrow \mathbb{R}$ be a qlsc bounded from above function and $s$ a winning strategy for the player $\alpha$ in the game $B M(X)$. Without loss of generality we may assume that the $s$-plays $\left\{U_{n}, V_{n}\right\}_{n}$ satisfy one additional requirement: $\bar{V}_{n} \subset U_{n}$ for every $n \geq 1$. We will construct by induction a winning strategy $\omega$ for the Player II in the game $B M(C(X), S(f) \cap M(f))$ in such a way that every $\omega$-play $\left\{W_{n}, W_{n}^{\prime}\right\}_{n \geq 1}$ will be accompanied by an $s$-play $\left\{U_{n}, V_{n}\right\}_{n \geq 1}$ and by two sequences $\left\{\varepsilon_{n}\right\}_{n \geq 1}$ and $\left\{\delta_{n}\right\}_{n \geq 0}$ of positive numbers so that the following requirements are satisfied for $i \geq 1$ :

$$
\begin{aligned}
& \overline{W_{i}^{\prime}} \subset W_{i} \text { and } \operatorname{diam}\left(W_{i}^{\prime}\right) \leq \delta_{i} \\
& S_{f}\left(W_{i}^{\prime}, \delta_{i}\right) \subset V_{i} \subset U_{i} \subset S_{f}\left(W_{i}, \varepsilon_{i}\right) \\
& \delta_{i} \leq \varepsilon_{i} / 4 \\
& \varepsilon_{i} \leq \delta_{i-1} / 2 .
\end{aligned}
$$

Let $\delta_{0}$ be some positive number. Let $W_{1}$ be an arbitrary open subset of $C(X)$. Take a closed ball $B\left[h_{1}, \varepsilon_{1}\right]$ with a $0<\varepsilon_{1} \leq \delta_{0} / 2$ which is contained in $W_{1}$. Consider the set $U_{1}:=$ int $S_{f}\left(h_{1}, \varepsilon_{1}\right)$ which, by condition (*), is a nonempty open set in $X$ and can be considered as a first legal move of the player $\beta$ in the game $B M(X)$. Set $V_{1}:=s\left(U_{1}\right)$. Since $\emptyset \neq V_{1} \subset$ $U_{1} \subset S_{f}\left(h_{1}, \varepsilon_{1}\right)$ we can apply Lemma 2.1 (b) to obtain $h_{1}^{\prime} \in C(X)$ and $\delta_{1} \leq \varepsilon_{1} / 4$ such that $B\left(h_{1}^{\prime}, \delta_{1} / 2\right) \subset B\left[h_{1}, \varepsilon_{1}\right] \subset W_{1}$ and $S_{f}\left(B\left(h_{1}^{\prime}, \delta_{1} / 2\right), \delta_{1}\right) \subset V_{1}$. Define $\omega\left(W_{1}\right):=W_{1}^{\prime}=$ $B\left(h_{1}^{\prime}, \delta_{1} / 2\right)$. We have $S_{f}\left(W_{1}^{\prime}, \delta_{1}\right) \subset V_{1}$. With this the first step in the induction process for the determination of the strategy $\omega$ is finished because the requirements (i), (ii), (iii) and (iv) are fulfilled for $i=1$. 
Suppose the strategy $\omega$ is defined up to the $n$-th stage of the game $B M(C(X), S(f) \cap M(f))$ so that each partial $\omega$-play $\left\{W_{i}, W_{i}^{\prime}\right\}_{1 \leq i \leq n}$ is accompanied by a partial $s$-play $\left\{U_{i}, V_{i}\right\}_{1 \leq i \leq n}$ and two finite sequences $\left(\varepsilon_{i}\right)_{1 \leq i \leq n}$ and $\left(\delta_{i}\right)_{0 \leq i \leq n}$ of positive numbers such that the requirements (i), (ii), (iii) and (iv) are satisfied for every $i, 1 \leq i \leq n$.

We will now extend the determination of the strategy $\omega$ to the next stage of the game. Let $\left\{W_{i}, W_{i}^{\prime}\right\}_{1 \leq i \leq n}$ be an arbitrary partial $\omega$-play and $W_{n+1}$ be any nonempty open subset of $W_{n}^{\prime}$. Take a function $h_{n+1} \in C(X)$ and some positive $\varepsilon_{n+1} \leq \delta_{n} / 2$ such that the closed ball $B\left[h_{n+1}, \varepsilon_{n+1}\right]$ is contained in $W_{n+1}$. By condition (*) the open set $U_{n+1}:=$ int $S_{f}\left(h_{n+1}, \varepsilon_{n+1}\right)$ is not empty. Because of the choice of $\varepsilon_{n+1}$ we have

$$
U_{n+1} \subset S_{f}\left(W_{n}^{\prime}, \delta_{n}\right) \subset V_{n} .
$$

Consider $U_{n+1}$ as a next choice of the player $\beta$ in the game $B M(X)$ at stage $n+1$. Set $V_{n+1}:=s\left(U_{1}, V_{1}, \ldots, V_{n}, U_{n+1}\right)$. Because $\emptyset \neq V_{n+1} \subset S_{f}\left(h_{n+1}, \varepsilon_{n+1}\right)$ we can apply Lemma 2.1 (b) to obtain a function $h_{n+1}^{\prime} \in C(X)$ and a positive number $\delta_{n+1} \leq \varepsilon_{n+1} / 4$ such that $B\left(h_{n+1}^{\prime}, \delta_{n+1} / 2\right) \subset B\left[h_{n+1}, \varepsilon_{n+1}\right]$ and $\left.S_{f}\left(B\left(h_{n+1}^{\prime}, \delta_{n+1} / 2\right), \delta_{n+1}\right)\right) \subset V_{n+1}$. We define the strategy $\omega$ at the stage $n+1$ of the game by

$$
\omega\left(W_{1}, W_{1}^{\prime}, \ldots, W_{n+1}\right):=W_{n+1}^{\prime}:=B\left(h_{n+1}^{\prime}, \delta_{n+1} / 2\right) .
$$

It follows from this construction that the properties (i), (ii), (iii) and (iv) are valid with $i=n+1$ as well. This finishes the determination of the strategy $\omega$.

Let $\left\{W_{n}, W_{n}^{\prime}\right\}_{n \geq 1}$ be an $\omega$-play with the accompanying $s$-play $\left\{U_{n}, V_{n}\right\}_{n \geq 1}$ and two tending to zero sequences $\left(\varepsilon_{n}\right)_{n \geq 1}$ and $\left(\delta_{n}\right)_{n \geq 0}$. Property (i) implies that the intersection $\cap_{n} W_{n}^{\prime}=\cap_{n} W_{n}$ is a singleton, say $h_{0} \in C(X)$. It follows from Lemma 2.1 (a) and (ii) that

$$
M_{f}\left(h_{0}\right)=\cap_{n} S_{f}\left(W_{n}^{\prime}, \delta_{n}\right)=\cap_{n} S_{f}\left(W_{n}, \varepsilon_{n}\right)=\cap_{n} U_{n}=\cap_{n} \overline{V_{n}} .
$$

This shows that $M_{f}\left(h_{0}\right)$ is a closed and nonempty set because $s$ is a winning strategy and $\cap_{n} U_{n}=\cap_{n} \overline{V_{n}} \neq \emptyset$. Thus $\left\{h_{0}\right\}=\cap W_{n}^{\prime} \subset S(f) \cap M(f)$ which according to the above mentioned result from the book of Oxtoby shows that $S(f) \cap M(f)$ contains a dense and $G_{\delta}$-subset of $C(X)$. The proof of the implication $(a) \Rightarrow(b)$ is completed.

The implication $(b) \Rightarrow(c)$ is trivial.

$(c) \Rightarrow(a)$. Suppose that for some qlsc bounded from above function $f: X \rightarrow \mathbb{R}$ we have $S(f) \supset \cap_{n} G_{n}$ where each $G_{n}, n \geq 1$, is open and dense in $C(X)$. We will construct by induction a winning strategy $s$ for the player $\alpha$ in the game $B M(X)$ in such a way that every $s$-play $\left\{U_{n}, V_{n}\right\}_{n \geq 1}$ is accompanied by a sequence $\left\{h_{n}\right\}_{n \geq 0}$ of functions from $C(X)$ and two sequences of positive integers $\left\{\varepsilon_{n}\right\}_{n \geq 0}$ and $\left\{\delta_{n}\right\}_{n \geq 1}$ such that, for every $i \geq 1$, the following requirements are fulfilled:

$$
\begin{aligned}
& V_{i}=\operatorname{int} S_{f}\left(h_{i}, \varepsilon_{i}\right) \subset S_{f}\left(B\left(h_{i}, \varepsilon_{i}\right), \delta_{i}\right) \subset U_{i} \\
& B\left[h_{i}, \varepsilon_{i}\right] \subset B\left(h_{i-1}, \varepsilon_{i-1}\right) \cap G_{i} \\
& \delta_{i} \leq \varepsilon_{i-1} / 4 \\
& \varepsilon_{i} \leq \delta_{i} / 2 .
\end{aligned}
$$

Take un arbitrary nonempty open set $U_{1}$ of $X$ which we consider as a first legitimate choice of player $\beta$ in the game $B M(X)$. Set $h_{0} \equiv 0$ and take some $x_{0} \in U_{1}$. Let $\varepsilon_{0}>0$ be such that $\varepsilon_{0}>\sup _{X} f-f\left(x_{0}\right) \geq 0$. Then $x_{0} \in S_{f}\left(h_{0}, \varepsilon_{0}\right) \cap U_{1}$ and we can apply Lemma 2.1 (b) to obtain $h_{1}^{\prime} \in C(X)$ and $\delta_{1}>0, \delta_{1} \leq \varepsilon_{0} / 4$, such that $S_{f}\left(B\left(h_{1}^{\prime}, \delta_{1} / 2\right), \delta_{1}\right) \subset U_{1}$ and $B\left(h_{1}^{\prime}, \delta_{1} / 2\right) \subset B\left(h_{0}, \varepsilon_{0}\right)$. Since $G_{1}$ is open and dense in $C(X)$ we can find $h_{1} \in C(X)$ and $\varepsilon_{1}<\delta_{1} / 2$ such that

$$
B\left[h_{1}, \varepsilon_{1}\right] \subset B\left(h_{1}^{\prime}, \delta_{1} / 2\right) \cap G_{1} .
$$


By condition (*) the set $V_{1}:=$ int $S_{f}\left(h_{1}, \varepsilon_{1}\right)$ is a nonempty open set. For it we have

$$
V_{1}=\text { int } S_{f}\left(h_{1}, \varepsilon_{1}\right) \subset S_{f}\left(B\left(h_{1}, \varepsilon_{1}\right), \delta_{1}\right) \subset S_{f}\left(B\left(h_{1}^{\prime}, \delta_{1} / 2\right), \delta_{1}\right) \subset U_{1} .
$$

Define the answer of player $\alpha$ under strategy $s$ to the first choice $U_{1}$ of player $\beta$ to be the set $s\left(U_{1}\right)=V_{1}=$ int $S_{f}\left(h_{1}, \varepsilon_{1}\right)$. This completes the first step in the induction process. The requirements (v), (vi), (vii) and (viii) are satisfied for $i=1$.

Suppose the strategy $s$ is defined up to the $n$-th stage of the game $B M(X)$ so that each partial $s$-play $\left\{U_{i}, V_{i}\right\}_{1 \leq i \leq n}$ is accompanied by a sequence $\left\{h_{i}\right\}_{0 \leq i \leq n}$ of functions from $C(X)$ and by two finite sequences $\left\{\varepsilon_{i}\right\}_{0 \leq i \leq n}$ and $\left\{\delta_{i}\right\}_{1 \leq i \leq n}$ of positive numbers such that the requirements (v), (vi), (vii) and (vii) are satisfied for every $i, 1 \leq i \leq n$. To extend the definition of $s$ to the next stage of the game, take an arbitrary nonempty open subset $U_{n+1}$ of $V_{n}=$ int $S_{f}\left(h_{n}, \varepsilon_{n}\right)$ and consider it to be the next move of player $\beta$. By Lemma 2.1 (b) there is $h_{n+1}^{\prime} \in C(X)$ and $\delta_{n+1}>0$ such that $\delta_{n+1} \leq \varepsilon_{n} / 4, S_{f}\left(B\left(h_{n+1}^{\prime}, \delta_{n+1} / 2\right), \delta_{n+1}\right) \subset U_{n+1}$ and $B\left(h_{n+1}^{\prime}, \delta_{n+1} / 2\right) \subset B\left(h_{n}, \varepsilon_{n}\right)$. Since $G_{n+1}$ is open and dense in $C(X)$, we can find $h_{n+1} \in C(X)$ and $\varepsilon_{n+1}>0$ such that $\varepsilon_{n+1}<\delta_{n+1} / 2$ and

$$
B\left[h_{n+1}, \varepsilon_{n+1}\right] \subset B\left(h_{n+1}^{\prime}, \delta_{n+1} / 2\right) \cap G_{n+1} \subset B\left(h_{n}, \varepsilon_{n}\right) \cap G_{n+1} .
$$
have

By condition $\left(^{*}\right)$ the set $V_{n+1}:=$ int $S_{f}\left(h_{n+1}, \varepsilon_{n+1}\right)$ is a nonempty open set. For it we

$$
V_{n+1} \subset S_{f}\left(B\left(h_{n+1}, \varepsilon_{n+1}\right), \delta_{n+1}\right) \subset S_{f}\left(B\left(h_{n+1}^{\prime}, \delta_{n+1} / 2\right), \delta_{n+1}\right) \subset U_{n+1} .
$$

Define the answer of player $\alpha$ under strategy $s$ to the choice $U_{n+1}$ of the player $\beta$ to be the set $s\left(U_{1}, V_{1}, \ldots, U_{n+1}\right):=V_{n+1}$. The induction is completed because the requirements (v), (vi), (vii) and (viii) are satisfied for $i=n+1$.

Every $s$-play $\left\{U_{n}, V_{n}\right\}_{n}$ in the game $B M(X)$ is accompanied by a sequence of functions $\left\{h_{n}\right\}_{n} \subset C(X)$ and by two sequences of positive numbers $\left\{\varepsilon_{n}\right\}_{n \geq 0}$ and $\left\{\delta_{n}\right\}_{n \geq 1}$ such that the requirements (v), (vi), (vii) and (viii) are satisfied.

We have the following two chains of equations and inclusions which have place for every $n \geq 1$ :

$$
U_{n+1} \subset V_{n}=\text { int } S_{f}\left(h_{n}, \varepsilon_{n}\right) \subset S_{f}\left(B\left(h_{n}, \varepsilon_{n}\right), \delta_{n}\right) \subset U_{n} .
$$

and

$$
B\left[h_{n}, \varepsilon_{n}\right] \subset B\left(h_{n-1}, \varepsilon_{n-1}\right) \cap G_{n} .
$$

By the latter and the fact that $\varepsilon_{n} \rightarrow 0$ the intersection $\cap_{n} B\left(h_{n}, \varepsilon_{n}\right)$ is a singleton, say $\left\{h^{*}\right\} \subset \cap_{n} G_{n}$. Hence, $M_{f}\left(h^{*}\right) \neq \emptyset$. The former chain of inclusions, the fact that $\varepsilon_{n} \rightarrow 0$ and Lemma 2.1 (a) imply that

$$
\emptyset \neq M_{f}\left(h^{*}\right)=\cap_{n} S_{f}\left(B\left(h_{n}, \varepsilon_{n}\right), \delta_{n}\right)=\cap_{n} V_{n} .
$$

Therefore $s$ is a winning strategy for the player $\alpha$ in the game $B M(X)$. The proof of the implication $(c) \Rightarrow(a)$, and thus of the theorem, is completed.

Remark 3.4 The proof of the implication $(a) \Rightarrow(b)$ of this theorem is, in essence, a transfer of a strategy from the game $B M(X)$ to a strategy for the game

$B M(C(X), S(f) \cap M(f))$. As every sequence $\left\{G_{n}\right\}_{n}$ of open and dense subsets of some topological space determines a strategy for Banach-Mazur game, the proof of the implication $(c) \Rightarrow(a)$ is also based on transfer of strategy (from the game $B M(C(X), S(f))$ to the game $B M(X)$ ). Without explicitly mentioning it, this idea of "strategy transfer" was used in [13], where the equivalence of $(a)$ and $(c)$ was proved for the partial case when $f \equiv 0$. In 
explicit and very refined form this idea is present in the paper of Debs and Saint Raymond [8] (again in connection with optimization problems with goal function $f \equiv 0$ ).

In optimization theory it is preferred to deal with well-posed problems. They are easier to solve, also numerically. This is why it is desirable to characterize the situations in which arbitrarily close to a given maximization problem there is a well-posed one. Given a bounded from above function $g: X \rightarrow \mathbb{R}$, the sequence $\left(x_{n}\right)_{n} \subset X$ is called maximizing for $g$, if $g\left(x_{n}\right) \rightarrow \sup _{X} g$. The function $g$ is said to have a strong maximum in $X$ if it has a unique maximizer $x_{0}$ in $X$ towards which converges every maximizing for $g$ sequence $\left(x_{n}\right)_{n}$. If $g$ has strong maximum in $X$, then the maximization problem $\max _{X} g$ is said to be well-posed.

Corollary 3.5 Let $X$ be a completely regular topological space. Then, the following statements are equivalent:

(a) There exists a winning strategy s for the player $\alpha$ in the game $B M(X)$ such that for every s-play $\left\{U_{n}, V_{n}\right\}_{n}$ the intersection $\cap_{n} U_{n}=\cap_{n} V_{n}$ is a singleton, say $\{x\}$, and the sequence $\left(V_{n}\right)_{n}$ is a local base for the point $x$ in $X$;

(b) For every qlsc bounded from above function $f: X \rightarrow \mathbb{R}$ the set $\{h \in C(X): f+h$ attains its strong maximum in $X\}$ contains a dense and $G_{\delta}$-subset of $C(X)$;

(c) There exists a qlsc bounded from above function $f: X \rightarrow \mathbb{R}$ for which the set $\{h \in C(X): f+h$ attains its strong maximum in $X\}$ contains a dense and $G_{\delta}$-subset of $C(X)$.

(d) The space $X$ contains a dense completely metrizable subset.

Proof $(a) \Rightarrow(b)$. Proceed as in the proof of the same implication in Theorem 3.3. Equation (3.2) implies that the maximizer $x$ of $f+h_{0}$ is unique while (3.1) shows that $V_{n}$ contains all but finitely many elements of every maximizing for $f+h_{0}$ sequence $\left(x_{n}\right)_{n}$. Hence the problem $\max _{X}\left(f+h_{0}\right)$ is well posed.

$(b) \Rightarrow(c)$ is evident.

$(c) \Rightarrow(a)$. Follow again the argument from the proof of the same implication in Theorem 3.3 to construct a strategy $s$ for player $\alpha$ in the game $B M(X)$. (3.4) and (3.5) show that $M_{f}\left(h_{0}\right)=\cap_{n} V_{n}$ is a singleton, say $x_{0}$, and this $x_{0}$ is the only maximizer of $f+h_{0}$ in $X$. It remains to prove that the sequence $\left(V_{n}\right)_{n}$ (or, equivalently, the sequence $\left.\left(U_{n}\right)_{n}\right)$ forms a neighborhood base at $x_{0}$. Suppose the contrary and take some open set $V \ni x_{0}$ such that, for every $n \geq 1$, there exists a point $x_{n} \in U_{n} \cap(X \backslash V)$. Then by (3.3) and $\left\{h_{0}\right\}=\cap_{n} B\left(h_{n}, \varepsilon_{n}\right)$ it follows that $\left(f+h_{0}\right)\left(x_{n}\right)>\sup _{X}\left(f+h_{0}\right)-\delta_{n}-3 \varepsilon_{n}$. Since $\varepsilon_{n}$ and $\delta_{n}$ tend to zero, we conclude that the sequence $\left(x_{n}\right)_{n}$ is maximizing for $f+h_{0}$ but does not converge to the maximizer $x_{0}$, a contradiction.

It follows from the equivalence of (b) and (c) that each of them is equivalent to the next statement (which is actually (c) for the function $f \equiv 0$ ):

$\left(c^{\prime}\right)$ The set $\{h \in C(X): h$ attains its strong maximum in $X\}$ contains a dense and $G_{\delta}$-subset of $C(X)$.

Using other methods (properties of special set-valued mappings) the equivalence of $\left(c^{\prime}\right)$ and (d) was established in Theorem 3.5 of [3]. An alternative direct proof that statements (a) and (d) are equivalent can be found in [5] (see Theorems 2.6 c) and 3.2 b)).

Remark 3.6 Topological spaces $(X, \tau)$ containing a dense completely metrizable subset appear in different mathematical contexts. In the just mentioned paper [5] it was proved that 
these and only these spaces admit a complete metric $d$ which is related to the topology $\tau$ in the following way:

i) The identity map $(X, d) \rightarrow(X, \tau)$ is continuous and

ii) For every nonempty $\tau$-open set $U$ and every $\varepsilon>0$ there exists a nonempty $\tau$-open set $V \subset U$ with $d-\operatorname{diam}(V)<\varepsilon$.

Related information about such spaces is contained also in [6].

The next remark concerns the validity of the above results in closed linear subspaces of $C(X)$.

Remark 3.7 Suppose that $Z$ is a closed linear subspace of $C(X)$ such that for every open set $U$ of $X$ and $x \in U$ there is a function $h \in Z, 0 \leq h(y) \leq 1$ for every $y \in X, h(x)=1$ and $\left.h\right|_{X \backslash U} \equiv 0$. Then, having in mind Remark 2.2 and also the proofs of Lemma 2.1 and Theorem 3.3 we conclude that the results in this section are true also restricted to the space $Z$.

\section{Some Applications}

It is well-known (and easy to check) that every lower semicontinuous function $f$ defined in a compact space $X$ attains its minimum. Such a function is not obliged to attain its maximum, even when it is bounded from above. Nevertheless, there are plenty of perturbations $h \in$ $C(X)$ for which the set of maximizers of $f+h$ is nonempty and compact.

Theorem 4.1 Let $f: X \rightarrow \mathbb{R}$ be a bounded from above qlsc function defined in the compact space $X$. Then the set $\left\{h \in C(X): M_{f}(h)\right.$ is nonempty and compact $\}$ contains a dense and $G_{\delta}$-subset of $C(X)$.

Proof In a compact space $X$ the player $\alpha$ has a simple winning strategy $s$ for the game $B M(X)$ : whenever player $\beta$ selects some $U_{n}$, player $\alpha$ responds by selecting some nonempty open set $V_{n}$ such that $\bar{V}_{n} \subset U_{n}$. For every $s$-play $\left\{U_{n}, V_{n}\right\}_{n}$ we have $\cap_{n} U_{n}=$ $\cap_{n} V_{n}=\cap_{n} \bar{V}_{n} \neq \emptyset$. It remains to apply Theorem 3.3.

Corollary 4.2 Let $f: X \rightarrow \mathbb{R}$ be a bounded from above qlsc function defined in the compact space $X$. Then the following properties are equivalent:

(a) The set $\{h \in C(X): f+h$ has unique maximizer in $X\}$ contains a dense and $G_{\delta^{-}}$ subset of $C(X)$;

(b) There exists a winning strategy $s$ for the player $\alpha$ in the game $B M(X)$ such that the intersection $\cap_{n} U_{n}=\cap_{n} V_{n}$ is a singleton for every s-play $\left\{U_{n}, V_{n}\right\}_{n}$;

(c) There exists a winning strategy s for the player $\alpha$ in the game $B M(X)$ such that, for every s-play $\left\{U_{n}, V_{n}\right\}_{n}$, the intersection $\cap_{n} U_{n}=\cap_{n} V_{n}$ is a singleton, say $\{x\}$, and the sequence $\left(V_{n}\right)_{n}$ is a local base for the point $x$ in $X$;

(d) The space $X$ contains a dense completely metrizable subset.

Proof We could use the reasoning from the proof of Theorem 3.3 to prove that (a) and (b) are equivalent. Suppose in $X$ there is a strategy $s$ with the property (b). Without loss of generality we may assume that the $s$-plays $\left\{U_{n}, V_{n}\right\}_{n}$ satisfy one additional requirement: 
$\bar{V}_{n} \subset U_{n}$ for every $n \geq 1$. Compactness of $X$ implies that, in this case, the sequence $\left(V_{n}\right)_{n}$ is a local base in $X$ for the point $\{x\}=\cap_{n} U_{n}=\cap_{n} V_{n}$. Hence (b) and (c) are equivalent.

The equivalence of (c) and (d) was discussed in the proof of Corollary 3.5.

We show next that the result from [11] mentioned in the Introduction is valid for qlsc functions as well.

Theorem 4.3 Let $(X, \rho)$ be a complete metric space and $B U C(X)$ be the space of all bounded uniformly continuous functions equipped with the sup-norm. Let $f: X \rightarrow \mathbb{R}$ be a bounded from above qlsc function. Then, the set $\{h \in B U C(X): f+h$ attains its strong maximum in $X$ \} contains a dense and $G_{\delta}$-subset of $B U C(X)$.

Proof For a complete metric space $(X, \rho)$ there is an obvious strategy $s$ with the property described in statement (a) of Corollary 3.5: whenever player $\beta$ selects some $U_{n}$, player $\alpha$ responds by selecting some nonempty open set $V_{n}$ such that $\bar{V}_{n} \subset U_{n}$ and $\rho-\operatorname{diam}\left(\bar{V}_{n}\right) \leq$ $n^{-1}$. On the other hand, if $U$ is un open subset of $X$, and $x \in U$, let $r>0$ be such that $B_{X}(x, r) \subset U$. The function $h: X \rightarrow[0,1]$ defined by $h(y):=1-\min \{\rho(x, y) / r, 1\}$, $y \in X$, is easily seen to be in $B U C(X)$ and satisfies $h(x)=1$ and $\left.h\right|_{X \backslash U} \equiv 0$. Since $B U C(X)$ is a closed linear subspace of $C(X)$, the result follows by Corollary 3.5 (b) and Remark 3.7

Corollary 4.4 If $f$ is a bounded lower semicontinuous function defined in the complete metric space $X$, then the set $\{h \in C(X): f+h$ attains both its strong maximum and its strong minimum in $X\}$ contains a dense and $G_{\delta}$-subset of $C(X)$.

Proof Theorem 3.5 from [4] implies that the set $\{h \in C(X): f+h$ attains its strong minimum in $X$ \} contains a dense and $G_{\delta}$-subset of $C(X)$. It follows from Corollary 3.5 of this paper that the $\operatorname{set}\{h \in C(X): f+h$ attains its strong maximum in $X\}$ also contains a dense and $G_{\delta}$-subset of $C(X)$. The intersection of the two dense and $G_{\delta}$-subsets of $C(X)$ is again such a set.

Acknowledgements First named author would like to acknowledge Robert Deville for inspirational input.

Open Access This article is licensed under a Creative Commons Attribution 4.0 International License, which permits use, sharing, adaptation, distribution and reproduction in any medium or format, as long as you give appropriate credit to the original author(s) and the source, provide a link to the Creative Commons licence, and indicate if changes were made. The images or other third party material in this article are included in the article's Creative Commons licence, unless indicated otherwise in a credit line to the material. If material is not included in the article's Creative Commons licence and your intended use is not permitted by statutory regulation or exceeds the permitted use, you will need to obtain permission directly from the copyright holder. To view a copy of this licence, visit http://creativecommons.org/licenses/by/4.0/.

\section{References}

1. Borwein, J.M., Preiss, D.: A smooth variational principle with applications to subdifferentiability and differentiability of convex functions. Trans. Amer. Math. Soc. 303, 517-527 (1987)

2. Čoban, M.M., Kenderov, P.S.: Dense Gâteaux differentiability of the sup-norm in $C^{*}(T)$ and the topological properties of T. Compt. Rend. Acad. Bulg. Sci. 38, 1603-1604 (1985) 
3. Čoban, M.M., Kenderov, P.S., Revalski, J.P.: Generic well-posedness of optimization problemsin topological spaces. Mathematika 36, 301-324 (1989)

4. Choban, M.M., Kenderov, P.S., Revalski, J.P.: Variational principles and topological games. Topol. Appls. 159(17), 3550-3562 (2012)

5. Choban, M.M., Kenderov, P.S., Revalski, J.P.: Fragmentability of open sets and topological games. Topol. Appl. 275, 107004 (2020). https://doi.org/10.1016/j.topol.2019.107004

6. Choban, M.M., Kenderov, P.S., Revalski, J.P.: Spaces with fragmentable open sets. Topol. Appl. 281, 107214 (2020). https://doi.org/10.1016/j.topol.2020.107214

7. De Blasi, F., Myiak, J.: Some generic properties in convex and nonconvex optimization theory. Ann. Soc. Math. Polon. 24, 1-14 (1984)

8. Debs, G., Saint Raymond, J.: Topological games and optimization problems. Mathematika 41, 117-132 (1994)

9. Deville, R., Godefroy, G., Zizler, V.: A smooth variational principle with applications to HamiltonJacobi equations in infinite dimensions. J. Funct. Anal. 111, 197-212 (1993)

10. Ekeland, I.: On the variational principle. J. Math. Anal. Appl. 47, 324-353 (1974)

11. Ivanov, M.: Perturbed maximisation of lower semicontinuous function, August 2018, https://doi.org/ 10.13140/RG.2.2.26992.20484

12. Kempisty, S.: Sur les fonctions quasi-continues. Fund. Math. 19, 184-197 (1932)

13. Kenderov, P.S., Revalski, J.P.: The Banach-Mazur game and generic existence of solutions to optimization problems. Proc. Amer. Math. Soc. 118, 911-917 (1993)

14. Kenderov, P.S., Revalski, J.P.: Dense existence of solutions of perturbed optimization problems and topological games. C. R. Acad. Bulgare Sci. 63(7), 937-942 (2010)

15. Lucchetti, R., Patrone, F.: Sulla densitàe genericità di alcuni problemi di minimimo ben posti. Boll. Un. Mat. Ital. B 15, 225-240 (1978)

16. Oxtoby, J.C.: The Banach-Mazur game and banach category theorem. In: Contributions to the theory of games, Vol. III, Annals of Math. Studies 39(Princeton 1957), pp 159-163 (1957)

17. Stegall, C.: Optimization of functions on certain subsets of Banach spaces. Math. Ann. 236, 171-176 (1978)

18. Ulam, S.M.: The Scottish Book, Los Alamos CA (1977)

Publisher's Note Springer Nature remains neutral with regard to jurisdictional claims in published maps and institutional affiliations.

\section{Affiliations}

\section{Ivanov ${ }^{1}$ P.S. Kenderov ${ }^{2}$ (D) . J.P. Revalski ${ }^{2,3}$}

M. Ivanov

milen@radiant-life-technologies.com

J.P. Revalski

revalski@math.bas.bg

1 Radiant Life Technologies Ltd., Nicosia, Cyprus

2 Institute of Mathematics and Informatics, Bulgarian Academy of Sciences, Acad. G. Bonchev str., block 8, 1113 Sofia, Bulgaria

3 Bulgarian Academy of Sciences, 1, 15th November str., 1040 Sofia, Bulgaria 